\title{
REPRESENTAÇÕES LÚDICAS DE ESTUDANTES DE ENFERMAGEM ACERCA DA SISTEMATIZAÇÃO DA ASSISTÊNCIA DE ENFERMAGEM
}

\author{
LUDIC REPRESENTATIONS OF NURSING STUDENTS ABOUT \\ THE SYSTEMATIZATION OF NURSING CARE
}

\section{REPRESENTACIONES LÚDICAS DE ESTUDIANTES DE ENFERMERÍA ACERCA DE LA SISTEMATIZACIÓN DE LA ATENCIÓN DE ENFERMERÍA}

\author{
Pétala Tuani Candido de Oliveira Salvador * \\ Kálya Yasmine Nunes de Lima ${ }^{* *}$ \\ Kisna Yasmin Andrade Alves ${ }^{* * *}$ \\ Cláudia Cristiane Filgueira Martins Rodrigues ${ }^{* * *}$ \\ Viviane Euzébia Pereira Santos ${ }^{* * * *}$
}

\begin{abstract}
RESUMO
Objetivo: Identificar as representações lúdicas de estudantes de Enfermagem acerca da Sistematização da Assistência de Enfermagem (SAE). Método: A coleta de dados foi realizada a partir de um grupo focal com oito estudantes de Enfermagem, utilizando a prática da mandala humanopoiética como técnica projetiva, através da representação figurativa com miniaturas e massas de modelar. O processo de análise de dados seguiu o rigor metodológico qualitativo, a partir do referencial metodológico da análise de conteúdo. Resultados: A representação lúdica dos estudantes perpassou três categorias de análise: benefícios da SAE; problemas vivenciados; e possibilidades de melhoria. Conclusão: Os estudantes representam positivamente a SAE, acreditando em sua consolidação. Destaca-se que o uso da mandala humanopoiética como técnica projetiva constitui um elemento qualificador do estudo, estimulando a expressão das subjetividades e favorecendo a verbalização de suas percepções.
\end{abstract}

Palavras chave: Planejamento de assistência ao paciente, processo de enfermagem, estudantes de Enfermagem.

\begin{abstract}
Objective: To identify the ludic representations of Nursing students about the Systematization of Nursing Care (SNC). Method: Data was collected from a focus group of eight Nursing students, using the human-poetic mandala as projective technique, through figurative representations with miniatures and modeling clay. A qualitative methodology for data analysis was used, based on content analysis as a referential method. Results: The

\footnotetext{
* Enfermeira. Docente da Escola de Saúde da Universidade Federal do Rio Grande do Norte. Natal, Brasil. Email: petalatuani@hotmail.com. Autor correspondente.

${ }^{* *}$ Enfermeira, Universidade Federal do Rio Grande do Norte. Natal, Brasil. Email: lima.yasmine@yahoo.com.br

${ }^{* * *}$ Enfermeira. Docente da Escola de Saúde da Universidade Federal do Rio Grande do Norte. Natal, Brasil. Email: kisnayasmin@hotmail.com

${ }_{* * * *}$ Enfermeira. Docente da Escola de Saúde da Universidade Federal do Rio Grande do Norte. Natal, Brasil. Email: claudiacrisfm@yahoo.com.br

${ }^{* * * * *}$ Enfermeira. Docente do Departamento de Enfermagem da Universidade Federal do Rio Grande do Norte. Natal, Brasil. Email: vivianeepsantos@gmail.com
} 
students' ludic representations included three thematic axes: benefits of the SNC; problems experienced; and possibilities for improvement. Conclusion: The students represented the SNC positively, believing in its consolidation. It is worth mentioning that the use of the human-poetic mandala as projective technique represents a qualifying element of the study, stimulating the expression of subjectivity and encouraging the verbalization of their perceptions.

Key words: Patient care planning, nursing process, nursing students.

\section{RESUMEN}

Objetivo: Identificar las representaciones lúdicas de estudiantes de Enfermería acerca de la Sistematización de la Atención de Enfermería (SAE). Material y método: La recolección de datos se realizó a partir de un grupo focal con ocho estudiantes de Enfermería de una universidad federal del Nordeste de Brasil, utilizándose la práctica de mandala humanopoiética como técnica proyectiva, a través de la representación figurativa con miniaturas y compuestos de modelado. El análisis de datos siguió el rigor metodológico cualitativo, a partir del referencial metodológico de análisis de contenido. Resultados: La representación lúdica de estudiantes impregnó tres temas: beneficios de la SAE; problemas experimentados y posibilidades de mejora. Conclusión: Los estudiantes representaron positivamente la SAE, creyendo en su consolidación. Cabe señalar que el uso de la mandala humanopoiética como técnica proyectiva es un elemento de calificación del estudio, pues estimula la expresión de subjetividades y fomenta la verbalización de percepciones.

Palabras clave: Planificación de atención al paciente, proceso de enfermería, estudiantes de enfermería.

Fecha recepción: 01/07/15 Fecha aceptación: 17/04/17

\section{INTRODUÇÃO}

A enfermagem, durante a sua história, perpassou por diversas mudanças relacionadas ao contexto sociopolítico em que estava inserida. A princípio, era uma atividade realizada por caridade, ligada à religião e sem muita produção científica no sentido de otimizar o cuidado prestado ao paciente. Diante disso, as práticas eram baseadas em empirismo e tradições (1).

Logo em seguida, a classe profissional passa a ser delineada pelos preceitos médicos e por eles determinada, configurando-se em uma profissão subordinada a classe médica e que se utilizava de seus conhecimentos para definir a sua prática $(1,2)$.

A partir da segunda metade do século XIX, contudo, a enfermagem começa um período de transição através do qual vem se desenvolvendo, passando por várias fases, até os tempos atuais. E como resultado, o cuidado passou a direcionar-se à recuperação e ao bem estar do indivíduo, com base num consistente conhecimento científico e na autonomia profissional (2).

A organização desse conhecimento científico ocorreu devido ao considerável avanço na construção e na organização dos modelos conceituais de enfermagem. Estes serviram como referencial para a elaboração das teorias de enfermagem, objetivando estabelecer uma relação entre diferentes conceitos, bem como melhor direcionar a assistência prestada ao ser humano $(1,2)$.

Essas teorias subsidiaram a edificação da Sistematização da Assistência de Enfermagem (SAE), a qual representa o instrumento de trabalho do enfermeiro com objetivo de identificar as necessidades do paciente, apresentando uma proposta ao seu atendimento 
e cuidado, direcionando a equipe de enfermagem nas ações a serem realizadas. Trata-se de um processo dinâmico e que requer na prática conhecimento técnico-científico, de modo que sua aplicabilidade é feita por meio do processo de enfermagem (3).

Contudo, embora o Conselho Federal de Enfermagem tenha tornado obrigatória a implementação da SAE nos serviços de saúde, ainda existem vários entraves à sua aplicação, que vão desde a formação profissional até a própria dinâmica das instituições de saúde $(3,4)$.

No que se refere à formação de enfermeiros, a literatura afirma a relevância do conhecimento teórico da SAE e sua aplicação ainda na universidade como facilitador para implantação na prática dos serviços de saúde. Torna-se imperativo, também, o preparo dos estudantes para lidar com as dificuldades do serviço, uma vez que apenas o aporte teórico não é suficiente para superar os problemas do cotidiano assistencial (1).

Outro fator relevante para contribuir com a consolidação da SAE seria uma maior articulação das instituições de formação com os serviços, o que pode ser otimizado através dos estudantes que usam esses serviços para suas aulas práticas e estágios, de modo que estes poderiam auxiliar e estimular o profissional do serviço a seguir esse modelo de assistência (1).

Nessa perspectiva, considerando a formação do profissional um elemento chave para a implementação da SAE, entende-se que identificar o que os estudantes do curso de graduação em Enfermagem compreendem acerca do tema pode contribuir para reflexão sobre a forma como esse conteúdo está sendo desenvolvido nas instituições formadoras, bem como para analisar possíveis lacunas existentes e a importância atribuída pelos estudantes à SAE, aspecto que influirá diretamente na sua prática profissional futura.

Deste modo, elencou-se como questionamento dessa pesquisa: como os estudantes do curso de graduação em enfermagem re- presentam ludicamente a SAE? Considera-se que investigar a representação lúdica dos estudantes possui relevância teórica, visto que “(...) a compreensão de como os alunos concebem sua futura profissão é necessária para o crescimento e fortalecimento da Enfermagem" (5).

Portanto, objetiva-se identificar as representações lúdicas de estudantes de enfermagem acerca da SAE.

\section{MATERIAL E MÉTODO}

O presente estudo corresponde a um recorte dos resultados da fase de ambientação com o campo e com o instrumento de dissertação de mestrado intitulada "Compreensão do típico ideal de técnicos de enfermagem acerca da sistematização da assistência de enfermagem", defendida pelo Programa de Pós-Graduação em Enfermagem da Universidade $\mathrm{Fe}$ deral do Rio Grande do Norte (UFRN), em 2013.

Trata-se de um estudo descritivo, de abordagem qualitativa, realizado com oito estudantes de enfermagem de uma universidade federal do Nordeste do Brasil. A coleta de dados foi realizada através da técnica do grupo focal, considerada uma técnica rápida e de baixo custo para avaliação e obtenção de dados e informações qualitativas (6).

O grupo focal foi realizado de acordo com a Pedagogia Vivencial Humanescente: realidade pedagógica transcorporal humanescente pautada nos saberes de dentro do Ser, de suas habilidades humanas, da sua subjetividade e da sua corporeidade (7), objetivando a expressão das motivações dos sujeitos.

Nesse contexto, foi utilizada a prática da mandala humanopoiética como técnica projetiva, através da representação figurativa com miniaturas e massas de modelar, para compreender as representações lúdicas dos estudantes de enfermagem sobre a SAE a partir da questão norteadora: "Quais são 
suas percepções acerca da SAE?".

Destaca-se que o uso de técnicas projetivas colabora para uma investigação de conteúdos inconscientes, propicia o diálogo e cria um ambiente favorável à investigação de aspectos subjetivos não revelados na verbalização, estimulando a expressão de sentimentos $(5,8)$.

Assim, a proposta consistia em utilizar a tríade "montar-falar-escrever" para estimular a expressão das percepções dos participantes acerca da questão norteadora: 1) montar um cenário; 2) falar acerca de suas representações, compartilhando ideias e opiniões; e 3) escrever a descrição do cenário construído, em um instrumento de pesquisa que contribuiu para a análise das falas dos estudantes.

O grupo focal, de acordo com a aquiescência dos estudantes, teve seu áudio gravado e contou ainda com um relator, que anotou aquilo que não pode ser captado pelas gravações, tais como: expressões faciais, expressões corporais, dentre outros aspectos; e de outro colaborador, responsável pelo registro fotográfico e apoio.

Além disso, o ambiente foi previamente preparado: no centro da sala estava o lugar onde a mandala foi construída, com um "tapete” de 3,00 x 4,20 m de Tecido Não Tecido (TNT) verde e um círculo central alaranjado, no qual estavam dispostas as tábuas de construção dos cenários, as massas de modelar e as miniaturas; em semicírculo, atrás do espaço anteriormente descrito, havia cadeiras, para que os participantes assistissem a apresentação da pesquisa e após assinassem os termos e preenchessem o questionário de caracterização; e, de frente para esses espaços, estava a cadeira em que a relatora ficou, descrevendo os detalhes percebidos no desenrolar do encontro.

O grupo focal, intitulado "O que eu penso acerca da SAE", totalizou 125 minutos e aconteceu em setembro de 2012, no Departamento de Enfermagem da Universidade Federal do Rio Grande do Norte (UFRN).

Inicialmente, a mediadora detalhou como seria a construção da mandala, momento em que os estudantes demonstraram ansiedade se conseguiriam realizar o proposto. $\mathrm{O}$ sentimento inicial de preocupação, entretanto, se transformou aos poucos em envolvimento com os cenários.

Os estudantes de enfermagem olhavam muitas vezes para a pergunta chave que estava projetada, refletindo sobre a melhor forma de representar suas percepções, analisando atentamente as miniaturas e manuseando as massinhas. Aos poucos, os cenários foram sendo construídos, com muita criatividade e reflexividade.

Após a construção das mandalas, os estudantes iniciaram o preenchimento do instrumento de descrição do cenário construído. Esta foi a etapa mais demorada, em que os estudantes elucidaram dúvidas quanto ao registro de suas produções, as quais foram retiradas pela mediadora.

Finalizando os registros, os estudantes de Enfermagem foram convidados a contemplar o cenário dos colegas para, posteriormente, sentar em frente a sua produção e iniciar o compartilhamento das vivências.

Após visualizar os cenários, os estudantes e a mediadora sentaram em círculo em volta das produções, formando a verdadeira mandala humanopoiética. Nesse momento, todos os estudantes puderam expor suas percepções, descrevendo seus cenários e respondendo a questão inicialmente proposta.

A própria equipe de pesquisa realizou a transcrição e leitura dos registros realizados pelo relator do grupo focal. Utilizou-se o Microsoft Word 2010 para a realização da transcrição do encontro e o Microsoft Excel 2010 para síntese dos resultados por meio de uma planilha.

As falas foram organizadas, mediante múltiplas leituras, o que possibilitou, juntamente com a análise dos cenários construídos, identificar as representações lúdicas dos estudantes de enfermagem acerca da SAE, processo que foi realizado a partir do referencial da análise de conteúdo temática (9). 
Desse modo, o processo de análise de dados seguiu o rigor metodológico qualitativo, aplicando-se os critérios de credibilidade e consistência teórico-epistemológica.

Os sujeitos da pesquisa foram identificados pelas letras EE (Estudante de Enfermagem), seguidas pelo numero sequencial de um a oito.

O estudo seguiu os princípios éticos e legais que regem a pesquisa científica em seres humanos, preconizados na Resolução ${ }^{\circ}$ 466/2012 do Conselho Nacional de Saúde, preservando o caráter voluntário dos participantes e o anonimato dos interlocutores, sendo aprovado pelo Parecer Consubstanciado do Comitê de Ética em Pesquisa da UFRN, no 98.424, de 31 de agosto de 2012, CAAE no 05906912.0.0000.5537.

\section{RESULTADOS}

O grupo focal foi realizado com a colaboração de oito estudantes de Enfermagem, de 19 a 24 anos, cursando o $5^{\circ}(3 ; 37 \%)$, o $6^{\circ}(2$; $26 \%$ ) ou o 7 o período $(3 ; 37 \%)$.

A partir da análise dos cenários, das falas e das descrições escritas dos estudantes de Enfermagem, foi possível compreender como que eles representavam ludicamente a SAE, o que, a partir da análise temática (9), permitiu emergir três categorias de análise: benefícios da SAE; problemas vivenciados; e possibilidades de melhoria.

\section{Benefícios da SAE}

Os estudantes de enfermagem, em sua totalidade, construíram uma imagem positiva da SAE, demonstrando que acreditam em sua essencialidade, o que pode ser reflexo de sua formação, cujo contato inicial com a temática se deu no $4^{\circ}$ período do curso, na disciplina Semiologia e Semiotécnica em Enfermagem.

Os benefícios da SAE foram representados ludicamente de diversas maneiras, com- binando miniaturas e massas de modelar, bem como utilizando palavras na construção de seus cenários.

Os bonecos de cores diferentes, por vezes unidos por suas mãos, foram utilizados por quase todos os estudantes para representar o cuidado individualizado e a aproximação do enfermeiro com o paciente, aspectos que podem ser alcançados com a solidificação da SAE. A fala de EE8 destaca o vínculo enfermeiro-paciente proporcionado pela SAE:

[...] a implantação da SAE ela meio que favorece o vinculo entre eles né, de forma que cria uma certa relação, porque à medida que você vai, como eu já falei, seguindo as etapas, você vai passo a passo, você vai se aproximando dele, você vai procurando entender o que está acontecendo, pra que assim possa desenvolver um cuidado eficaz né [...] (EE8).

Destacou-se, ainda, que a SAE, à medida que permite tal visão individualizada das necessidades e subjetividades dos usuários, permite a solidificação de um cuidado humanizado, representado, pelos estudantes de enfermagem, por brinquedos (peão, carrinho, dentre outros) e por flores, demonstrando a sensibilidade que deve existir no cuidado do enfermeiro, para enxergar as individualidades de seus pacientes.
A flor significa a humanização, pois o profis- sional não olhará somente para as queixas do paciente e tratar a patologia, irá conversar para colher a anamnese, tocar para fazer o exame fisico, orientar para traçar os resultados e, aci- ma de tudo, cuidar do paciente, assim como flor precisa para viver, sendo regada, podada, tendo atenção (EE7).

Em outros cenários, o reconhecimento profissional foi representado por um avião, indicando os voos que a enfermagem pode alçar, por escadas construídas de peças de lego, bem como por patinhos unidos, indicando que tal valorização só poderá ser alça- 
da por meio de um trabalho em equipe. O uso da palavra REGISTRO no cenário de EE3 segue o mesmo pensamento:

O REGISTRO dos cuidados realizados proposto pela SAE de forma sistematizada também é uma forma de promover o crescimento da profissão, pois possibilita aos demais reconhecer/ perceber a atuação da enfermagem (EE3).

O uso de letras e palavras também apareceu em outros cenários para representar benefícios da SAE: as letras COM indicando a comunicação entre a equipe; a palavra ATUALIZAÇÃO representando o crescimento profissional; as letras $\mathrm{E}$ e $\mathrm{C}$ mostrando que a SAE resulta da junção dos conhecimentos empíricos e científicos para organizar o cuidado; e as letras CDPIA como meio de representar as iniciais das etapas do processo de enfermagem - coleta de dados, diagnóstico, prescrição, implementação e avaliação.

O relógio representou, para a maioria dos estudantes de Enfermagem, a otimização do tempo possibilitada pela SAE:

[...] com a sistematização né, o próprio nome já diz, vai sistematizar o trabalho do enfermeiro $e$ com isso ele vai economizar tempo, isso vai otimizar, só que pra isso é preciso que as pessoas percebam isso. Enquanto elas não perceberem que no início você vai requerer um tempo maior, mas depois isso vai ser satisfatório (EE5).

O relógio também vai dar o sentido no tempo e no espaço pras pessoas que utilizam e o sentido da profissão, que seria a organização e a cronicidade das etapas que o enfermeiro irá seguir (EE7).

\section{Problemas vivenciados para concretizar a SAE}

Os estudantes representaram a partir da letra $\mathrm{X}$ construída em massa de modelar vermelha o que visualizam como cenário atual da SAE: benefícios que se contrapõem a dificuldades inerentes à prática de enfermagem, mas que podem ser superadas por meio, sobretudo, da crença e na busca da efetivação da SAE.

O relógio, que em todos os outros cenários apareceu como elemento que representava um benefício da SAE - a otimização do tempo, é destacado no cenário de EE1 como um fator dificultador: a falta de tempo dos profissionais de saúde.

O peão, ao lado do relógio, destaca que os profissionais de enfermagem são exigidos quanto à rapidez e agilidade de sua prática profissional, o que acaba por ser um entrave para a consolidação efetiva da SAE.

Outros cenários destacaram que a sobrecarga de trabalho e as más condições nos ambientes laborais contribuem para que a SAE não seja efetivada, aspecto que foi representado por um boneco rodeado de soldados, representando todos os elementos dificultadores presentes no ambiente de trabalho da equipe de enfermagem.

Adiciona-se, ainda, o despreparo e o desconhecimento de muitos profissionais acerca da SAE, o que foi representado por EE1 como um jarro vazio - a falta de conhecimento. EE4 também representou tal aspecto, por meio de uma peça de quebra-cabeça:

a SAE ainda é um quebra-cabeça para os profissionais, porque muitos não tem conhecimento e não sabem como implementá-la (EE4).

Os estudantes de enfermagem enfatizaram o distanciamento do profissional de enfermagem no que se refere à interação com o paciente como problemas vivenciados, representando ludicamente esse entrave por meio de bonecos distanciados. O terço presente no cenário de EE1 elucida tal questão, apontando que muitos profissionais tem dificuldade de compreender as individualidades dos pacientes.

Como problemas, eu trouxe uma barreira que existe muitas vezes entre os profissionais da saúde, onde um se acha superior ao outro, e isso 
complica, dificulta a sistematização, e também uma barreira entre os profissionais de saúde e o paciente, que muitas vezes os profissionais de saúde não conseguem estabelecer um elo adequado com o paciente e acaba deixando pela metade a assistência que o paciente necessita (EE2).

É válido destacar, ainda, uma reflexão trazida por EE8, que compreende que a predominância do modelo biomédico constitui o elemento gerador dos entraves vivenciados e relatados, uma vez que dificulta a edificação do raciocínio clínico, do cuidado individualizado, reflexivo e humanizado:

[...] agem de modo igual com todos os pacientes, sem ver as suas particularidades; além de exercerem a enfermagem sem um raciocínio clínico, seguindo de modo tecnicista (EE8).

\section{Possibilidades de melhoria: o caminho a trilhar}

Os estudantes acreditam que, para consolidar a SAE, é necessário: perseverança e luta dos profissionais (representadas ora por soldados ora por bonecos unidos); trabalho em equipe (palavras FOCO, patinhos e avião); atualização e implementação do conhecimento necessário (livro e jarro com flor compreendendo a terra como o conhecimento necessário e a flor como a sensibilidade do cuidado implementado); e um ambiente de trabalho adequado (flores).

Para melhorar o quadro de dificuldades vivido, faz-se necessário agilidade do profissional, foco para manter seus objetivos, união da equipe e interação com o paciente, ambiente adequado para desenvolver as atividades e conhecimento do seu cliente como um todo para atendê-lo com qualidade e de forma integral. Além da atualização de conhecimento científico por parte do enfermeiro, procurando aperfeiçoar-se cada vez mais (EE4).
Os estudantes de Enfermagem defendem, desse modo, que, mesmo com os entraves vivenciados pela profissão, a SAE pode ser implementada. Isso é representado por EE7, quando representa em seu cenário uma escada, em que, em seu topo, está um bebê:

[...] o caminhar da enfermagem pela SAE. Cada degrau vai ter sua dificuldade, mas ao final dessa escada a gente tem o quê? Como se fosse a vitória. E por que o bebê? O bebê simboliza a vida, simboliza nascimento, então pra mim esse bebê simboliza o nascimento e a profissão cada vez mais aprimorada, cada vez melhor. Então cada etapazinha vai fazer com que a enfermagem se torne cada vez melhor, qualificada e humanizada (EE7).

\section{DISCUSSÃO E CONCLUSÃO}

Observou-se que o uso da mandala como técnica projetiva mostrou-se efetivo, uma vez que contribuiu, de forma semelhante a outros estudos que utilizaram representações lúdicas como forma de coleta de dados, para que a verbalização dos sentimentos e de subjetividades fosse alçada $(5,8,10)$.

Os benefícios da SAE destacados pelos estudantes de Enfermagem são corroborados pela literatura, que destaca que o usuário, foco dos benefícios da SAE, também é influenciado positivamente, uma vez que a SAE possibilita maior confiança, satisfação, gratidão, melhor recuperação e redução da ansiedade do cliente (11).

Desse modo, à medida que a SAE focaliza o cuidado individualizado e humanístico, ela também proporciona a validação das funções da enfermagem, contribuindo para o reconhecimento da importância das ações de enfermagem em qualquer nível de assistência à saúde e aumentando sua autonomia e visibilidade, criando fenômenos com características próprias e específicas para o cuidado, 
essência da profissão (12-14).

Outrossim, os estudantes de enfermagem também enfatizaram que a SAE traz como benefícios o crescimento profissional, o reconhecimento e a valorização da área de atuação da enfermagem, isso por meio da visibilidade do cuidado como a essência da profissão.

Dessa forma, visualiza-se a SAE como uma metodologia empregada na organização do conhecimento e do cuidado ao usuário, constituindo uma atividade intelectual deliberada, caracterizando-se por ser intencional, sistemática, dinâmica, interativa, flexível e baseada em teorias (15).

Os estudantes enfatizaram a otimização do tempo possibilitada pela SAE, aspecto destacado também pela literatura, que considera como vantagens dessa metodologia de trabalho: a maior segurança na tomada de decisões, a economia de tempo e a facilidade de planejamento da assistência, diminuindo, inclusive, o tempo de internação hospitalar $(12,16)$.

Outros cenários destacaram ainda que a SAE proporciona à equipe de enfermagem $o$ desenvolvimento de um raciocínio clínico, através da formação de dúvidas e hipóteses (representadas por um ponto de interrogação) e do fomento de novas ideias (um ponto de exclamação), o que vai favorecer a qualificação do cuidado na medida em que este estará norteado por conhecimentos científicos.

Destarte, de maneira semelhante a um estudo que buscou, por meio da técnica do desenho projetivo, identificar as percepções de recém-admitidos ao curso de graduação em enfermagem de uma universidade pública do Ceará acerca da profissão, elucidou-se que os estudantes de enfermagem representaram ludicamente uma imagem profissional valorizada, com base em novas perspectivas que privilegiam a verdadeira essência da Enfermagem: o cuidado à pessoa humana (5).

Todavia, ao mesmo tempo em que os estudantes de enfermagem reconhecem a essencialidade da SAE enquanto elemento qualificador da prática profissional, apontam que essa não é efetivada nos ambientes assistenciais, refletindo suas vivências em estágios, em que não visualizaram, em momento algum, tentativas ou efetivações da SAE.

A literatura destaca que, ao se deparar com inúmeras dificuldades, a desmotivação profissional é visualizada, contribuindo para o fato de o trabalho sistematizado ainda não estar incorporado à prática assistencial, estando mais presente no discurso dos profissionais, o que se traduz num enfraquecimento e numa desarticulação da teoria com a prática, fato este que gera conflitos ideológicos que prejudicam não somente o entendimento da prática de enfermagem, como também o ensino das teorias de enfermagem, do processo de enfermagem e da $\operatorname{SAE}(11,17)$.

Em grande parte, o desconhecimento, a dificuldade de domínio técnico-científico e a resistência dos profissionais, por entender a SAE como uma atividade burocrática que os distancia do usuário, são vistos como elementos dificultadores $(11,14,18)$.

O desconhecimento, sobretudo, é visto como reflexo de uma formação que ainda privilegia habilidades técnicas: resultados de um estudo, que entrevistou 27 enfermeiros do hospital universitário (HU) da Universidade Federal de Sergipe (UFS), demonstraram que $63 \%$ dos entrevistados afirmaram terem estudado somente a teoria do PE e não o terem aplicado (ou aplicado superficialmente) na prática, enquanto que $18,5 \%$ referiram não ter estudado ou não lembraram de ter sido abordado este assunto na sua formação (19).

Os estudantes de enfermagem ainda enfatizaram os conflitos entre os profissionais de saúde e o distanciamento do profissional de enfermagem no que se refere à interação com o paciente como problemas vivenciados.

A SAE, destarte, é elucidada como uma ferramenta de empoderamento das práticas de enfermagem, sem a qual o enfermeiro se resume a um papel burocrático -o denominado "enfermeso"- executando um mero fa- 
zer, reflexo do modelo biomédico que fragmenta o processo de cuidar, comprometendo a qualidade da assistência, desvalorizando-se enquanto profissional $(11,19)$.

À medida que refletiram sobre as dificuldades vivenciadas para a real consolidação da $\mathrm{SAE}$, os estudantes de enfermagem apontaram caminhos que devem ser trilhados como meio de superação dos entraves e afirmação dos benefícios.

Entende-se, dessa forma, que desafios precisam ser superados, compreendendo que a implementação da SAE exige da profissão uma integração efetiva com a equipe multidisciplinar.

Além disso, o planejamento da efetivação da SAE deve sofrer as adequações necessárias a cada contexto institucional, isso através do levantamento do sistema como um todo valores, recursos humanos e suas funções, estrutura política de gestão institucional, capacitação profissional, necessidades específicas/ perfil dos usuários, sensibilização e preparo de toda a equipe de enfermagem, definição do referencial teórico, elaboração de instrumentos e preparo prático para a implementação da SAE (15-19).

Nesse ínterim, considera-se que a SAE pode contribuir para o resgate do poder da enfermagem à medida que valoriza o cuidado como essência da profissão, assumindo características de prática reflexiva, transformando o modo de pensar para uma mudança que busca a qualidade do cuidar.

Apreende-se que os estudantes acreditam na SAE como elemento qualificador da prática de enfermagem, proporcionando como benefícios a solidificação de um cuidado individualizado, reflexivo, sistematizado e humanizado, o que contribui essencialmente para o reconhecimento profissional e para a valorização do cuidado como essência da enfermagem.

Reconheceu-se, entretanto, que muitos entraves ainda integram o ambiente de trabalho dos profissionais de enfermagem, como a sobrecarga de trabalho e as más condições laborais, o que, somado ao desconhecimento e à predominância do pensamento fragmentado, dificulta a efetivação da SAE.

Contudo, reconhecendo seus benefícios e defendendo a implementação da SAE, os estudantes de enfermagem traçaram possibilidades de melhoria, na forma de um caminho a ser trilhado pela enfermagem, envolvendo união, perseverança, lutas por melhores condições de trabalho e a busca incessante de conhecimento.

Destaca-se que o uso da mandala humanopoiética como técnica projetiva constituiu um elemento qualificador do estudo, estimulando a expressão das subjetividades e favorecendo a verbalização das percepções. Acrescenta-se, ainda, que, ao unir a representação por meio de cenários, a descrição escrita dos mesmos e o compartilhamento verbal das construções, a expressão das representações lúdicas foi enriquecida.

É válido destacar que os resultados apresentados constituem a expressão de uma realidade específica, em que a formação dos estudantes de enfermagem influenciou positivamente em suas percepções positivas acerca da SAE.

Espera-se, desse modo, contribuir para que outros estudos sejam alçados, uma vez que conhecer a opinião de estudantes de enfermagem significa compreender como que o ensino está sendo refletido na formação crítica e reflexiva de futuros profissionais, que irão lutar pela consolidação efetiva na SAE.

\section{REFERÊNCIAS}

1. Santos WN. Sistematização da assistência de enfermagem: o contexto histórico, o processo e obstáculos da implantação. J Manag Prim Health Care. 2014; 5(2): 153-158.

2. Benedet SA, Gelbcke FL, Amante LA, Padilha MIS, Pires DP. Processo de enfermagem: instrumento da sistematização 
da assistência de enfermagem na percepção dos enfermeiros. Rev. pesqui. cuid. fundam. (Online) [Internet]. 2016 [citado 20 ago 2017]; 8(3): 4780-4788. Disponível em: http://pesquisa.bvsalud.org/ bvsvs/resource/pt/bde-28758

3. Schmitz EL, Gelbcke FL, Bruggmann MS, Luz SCL. Filosofia e marco conceitual: estruturando coletivamente a sistematização da assistência de enfermagem. Rev Gaucha Enferm [Internet]. 2016 [citado 20 ago 2017]; 37 (esp): e68435. Disponível em: http://www.scielo.br/scielo.php?pi$\mathrm{d}=$ S1983-14472016000500405\&script $=$ - ci_abstract

4. Silva RS, Almeida ARLP, Oliveira FA, Oliveira AS, Sampaio MRFB, Paixão GPN. Sistematização da Assistência de Enfermagem na perspectiva da equipe. Enferm. Foco. 2016; 7(2): 32-36.

5. Sousa FEM, Oliveira EN, Nunes JM, Lopes RE, Gubert FA. Percepção de estudantes de enfermagem acerca da profissão. Rev. Rene. 2010; 11(4): 110-117.

6. Minayo MCS. O desafio do conhecimento: pesquisa qualitativa em saúde. $13^{\mathrm{a}} \mathrm{ed}$. São Paulo (SP): Hucitec; 2013. 407 p.

7. Cavalcanti KB (org.). Pedagogia Vivencial Humanescente: para sentirpensar os sete saberes na educação. Curitiba (PR): Editora CRV; 2010. 206 p.

8. Teixeira MA, Paiva MS, Nóbrega SM, Nitschke RG. Manuseio com massa de modelar: uma estratégia sensível de coleta de dados na pesquisa em saúde e enfermagem. Texto contexto - enferm. 2013; 22(3): 857-863.

9. Bardin L. Análise de conteúdo. São Paulo (SP): Edições 70; 2011. 279 p.

10. Hirt MC, Costa MC, Arboit J, Leite MT, Hesler LZ, Silva EB. Representações sociais da violência contra mulheres rurais para um grupo de idosas. Rev Gaucha Enferm [Internet]. 2017 [citado 30 dic 2017]; 38(4): e68209. Disponível em: http://www.seer.ufrgs.br/index.php/
RevistaGauchadeEnfermagem/article/ view/68209

11. Krauzer IM, Adamy EK, Ascari RA, Ferraz L, Trindade LL, Neiss M. Sistematização da assistência de enfermagem na atenção básica: o que dizem os enfermeiros? Cienc. enferm. 2015; XXI(2): 31-38.

12. Gonçalves MRCB, Spiri WC, Ortolan EVP. Compreensão dos enfermeiros gerentes sobre o processo de enfermagem. Cienc Cuid Saude. 2016; 15(2): 336-342.

13. Silva TG, Santos RM, Crispim LMC, Almeida LMWS. Conteúdo dos registros de enfermagem em hospitais: contribuições para o desenvolvimento do processo de enfermagem. Enferm. Foco. 2016; 7(1): 24-27.

14. Soares MI, Resck ZMR, Terra FS, Camelo SHH. Sistematização da assistência de enfermagem: facilidades e desafios do enfermeiro na gerência da assistência. Esc Anna Nery Rev Enferm. 2015; 19(1): 4753.

15. Rosa CS da, Lunardi Filho WD, Pimpão FD, Vettorello JS. O ensino do processo de enfermagem na ótica dos docentes. Rev enferm UFPE on line [Internet]. 2015 [citado 30 ago 2017]; 9(6): 82358244. Disponível em: https://periodicos. ufpe.br/revistas/revistaenfermagem/article/view/10583/11535

16. Souza NR, Costa BMB, Carneiro DCF, Barbosa HSC, Santos ICRV. Sistematização da assistência de enfermagem: dificuldades referidas por enfermeiros de um hospital universitário. Rev enferm UFPE on line [Internet]. 2015 [citado 30 ago 2017]; 9(3): 7104-7110. Disponível em: https://periodicos.ufpe.br/revistas/revistaenfermagem/article/view/10440/11246

17. Gutiérrez MGR, Morais SCRV. Systematization of nursing care and the formation of professional identity. Rev Bras Enferm. 2017; 70(2): 436-441.

18. Massaroli R, Martini JG, Massaroli A, Lazzari DD, Oliveira SN, Canever BP. Traba- 
lho de enfermagem em unidade de terapia intensiva e sua interface com a sistematização da assistência. Esc Anna Nery Rev Enferm. 2015; 19(2): 252-258.
19. Andrade JS, Vieira MJ. Prática assistencial de enfermagem: problemas, perspectivas e necessidade de sistematização. Rev Bras Enferm. 2005; 58(3): 261-265. 